\title{
FOLTER UND MENSCHENRECHTE IM INTERDISZIPLINÄREN RAHMEN
}

\author{
Thomas WeNzeL, World Psychiatric Association, Scientific Section on \\ Psychological Aspects of Torture and Persecution and \\ CEHRI
}

Reem AlKsIRI, World Psychiatric Association, Scientific Section on Psychological Aspects of Torture and Persecution and Syrian Centre for Legal Studies and Research

\section{INHALT}

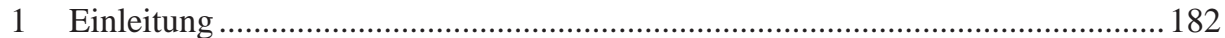

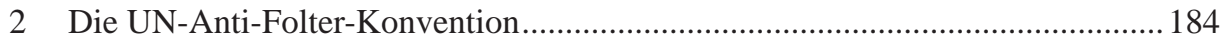

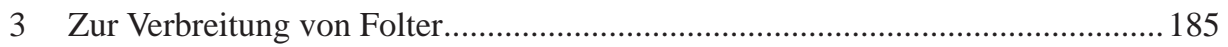

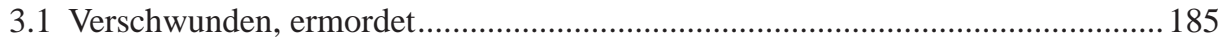

3.2 Folter als Teilfaktor von Verfolgungshandlungen ................................................. 186

3.3 Voraussetzungen der Folter und schwerwiegender Menschenrechtsverletzungen - individual- und gruppendynamische Aspekte......................186

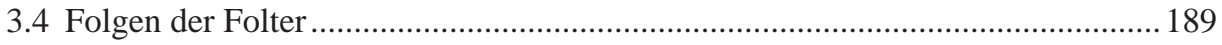

4 Aspekte der Behandlung .................................................................................. 191

4.1 Angehörige und Familien als indirekte Folteropfer ................................................. 191

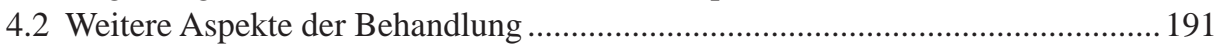

4.3 Transitional Justice - Therapeutic Justice........................................................... 192

4.4 Zur Schnittstelle Medizin, Psychologie und Recht................................................. 193

4.5 Die Rolle von Gesundheitsberufen im Bereich des Menschenrechtsschutzes und der Folterprävention ..............................................193

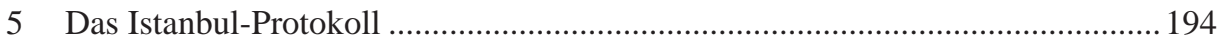

5.1 Das Istanbul-Protokoll im Asylverfahren.............................................................196

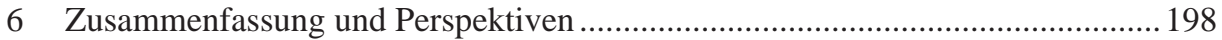

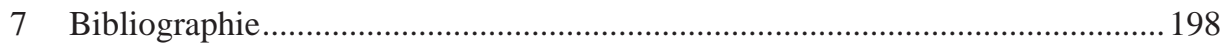




\section{Einleitung}

Spätestens seit den traumatischen Erfahrungen des Zweiten Weltkriegs und den Nürnberger Prozessen wurde deutlich, dass grundlegende Konzepte des humanitären Völkerrechts und der Menschenrechte, die teilweise bereits infolge des Krimkrieges in Bezug auf Krieg führende Parteien festgelegt worden waren, einer grundlegenden Revision und Ausweitung besonders in Bezug auf Zivilist/inn/en bedurften (McCoubreY 1995).

Dies wurde in einem ersten Schritt in der Allgemeinen Erklärung der Menschenrechte realisiert, und kurz darauf folgten die erweiterten Genfer Konventionen mit dem Schwerpunkt des humanitären Völkerrechts sowie der Zivilpakt ${ }^{1}$ (1966), der sich ebenfalls mit Grundprinzipien der Menschenrechte beschäftigt. ${ }^{2}$ Eine grundliegende Überlegung war dabei, dass schwerwiegende Verbrechen, insbesondere Verbrechen gegen die Menschlichkeit, aber auch grundlegende Freiheiten nicht erst durch lokale Gesetze behandelt werden können und somit der Willkür von Machthabern unterworfen sein dürfen, sondern dass es in diesem Bereich um grundlegende Aspekte der Zivilisation geht, deren Notwendigkeit für jeden einleuchtend sein sollte. Daher seien diese auch als stillschweigendes Recht aller Nationen (also jus cogens) (DE WET 2004) (oder natürliches Recht „natural law“) grundlegend und für jeden Menschen zu gewährleisten.

In der Folge wurden einige grundlegende, besonders wichtige Ansprüche, wie beispielsweise das in der allgemeinen Deklaration der Menschenrechte ${ }^{3}$ und in folgenden Erklärungen und Konventionen angeführte absolute Folterverbot, unter besonderen Schutz gestellt, indem diese als unaufhebbar („non-derogable“) eine Sonderstellung erhielten. Diese beinhaltet, dass sie im Gegensatz zu einigen anderen Menschenrechten nie und mit keiner wie auch immer gearteten Begründung aufgehoben oder eingeschränkt werden dürfen. Folter wird trotz dieses Grundsatzes weltweit und eher zunehmend angewandt (BuscH et al. 2015), und ist einerseits für Jurist/inn/en und die Zivilgesellschaft die wichtigste „Waffe gegen Demokratie“, und für Ärzte/innen, Psycholog/inn/en und Psychotherapeut/inn/en aufgrund ihrer schweren Langzeitfolgen bei unmittelbar Betroffenen wie auch bei indirekten Opfern von hoher Relevanz (DE Wet 2004; Furtmayr \& Frewer 2010). In einem interdisziplinären Rahmen sind dabei sowohl juristische und menschenrechtliche als auch soziologische, politische, kulturanthropologische, psychologische und medizinische Faktoren sowohl für die Prävention wie auch für die Rehabilitation und Behandlung zu berücksichtigen (WEnzel, Frewer et al. 2015) und werden daher in den folgenden Artikel einbezogen. Es ist dabei anzumerken, dass viele auch grundlegende Menschenrechte oft einer Diskussion ihrer kulturabhängigen Anwendbarkeit und Kultursensitivität ausgesetzt worden sind (MusALO 1997), was allerdings für das absolute Folterverbot in der Praxis, außer in fragwürdiger

$1 \quad$ Vgl. $<$ https://www.zivilpakt.de/> [Zugriff: 21-01-2020].

2 Vgl. <https://www.ohchr.org/Documents/Publications/training8Revlen.pdf $>$, Chapter 1. [Zugriff: 21-01-2020].

$3 \quad$ Vgl. <https://www.menschenrechtserklaerung.de/> [Zugriff: 21-01-2020]. 
Weise in Bezug auf den „Krieg gegen Terror“ (SingH 2007; Moon 2004), so gut wie nicht gilt.

Während anfangs Folter nicht immer gleich definiert wurde - so gibt es beispielsweise eine weitere Definition des Begriffs Folter in der ursprünglichen Deklaration des Weltärzteverbandes -, hat es sich inzwischen zunehmend durchgesetzt, konsequent die Definition der UN-Deklaration gegen Folter („Antifolterdeklaration“, 4 1984) als Referenz zu berücksichtigen oder sich auf diese zu beziehen:

„,... jede Handlung, durch die einer Person vorsätzlich große körperliche oder seelische Schmerzen oder Leiden zugefügt werden, zum Beispiel um von ihr oder einem Dritten eine Aussage oder ein Geständnis zu erlangen, um sie für eine tatsächlich oder mutmaßlich von ihr oder einem Dritten begangene Tat zu bestrafen, um sie oder einen Dritten einzuschüchtern oder zu nötigen oder aus einem anderen, auf irgendeiner Art von Diskriminierung beruhenden Grund, wenn diese Schmerzen oder Leiden von einem Angehörigen des öffentlichen Dienstes oder einer anderen in amtlicher Eigenschaft handelnden Person, auf deren Veranlassung oder mit deren ausdrücklichem oder stillschweigendem Einverständnis verursacht werden. Der Ausdruck umfasst nicht Schmerzen oder Leiden, die sich lediglich aus gesetzlich zulässigen Sanktionen ergeben, dazu gehören oder damit verbunden sind. " 5

Wichtig ist dabei auch die Erweiterung der Definition des Schwerpunkts der Konvention um den Tatbestand der „unmenschlichen und erniedrigenden Behandlung“, die dem gleichen absoluten Verbot unterliegt und in der Folge gleichwertig behandelt wird. Sie unterscheidet sich von Folter im engeren Sinne dadurch, dass die Intentionalität nicht vorliegt oder im Hintergrund steht.

Folter selbst dient dabei, wie die Literatur der letzten Jahrzehnte gezeigt hat, unterschiedlichen Zielen, und meist nicht - wie man annehmen könnte - dem Gewinnen von Informationen, wozu sie im Weiteren auch nicht geeignet ist, wie verschiedene Autor/ inn/en zu Recht argumentiert haben (WitZLING 2015). Von Täter/inne/n angegebene „Ziele“ von Folter sind vor allem:

- Informationsgewinnung,

- die Aufklärung oder Verhinderung von Straftaten,

- das Erpressen eines Geständnisses,

- Terror gegen Individuen, Gruppen oder Angehörige,

- finanzielle Erpressung,

- Einschüchterung von Zeugen sowie

- individuelle, sadistische Ziele der Täter/innen.

$4 \quad$ Vgl. $<$ https://www.antifolterkonvention.de/> [Zugriff: 21-01-2020].

$5 \quad$ Vgl. $<$ https://www.antifolterkonvention.de/definition-der-folter-3153/> [Zugriff: 21-01-2020]. 


\section{Die UN-Anti-Folter-Konvention}

Die UN-Anti-Folter-Konvention (CAT) bedarf eigentlich der Ratifizierung durch ein Land, wobei die Mehrheit der UN Mitgliedstaaten inzwischen die Konvention unterzeichnet hat: ${ }^{6}$ Das absolute Folterverbot wurde auch in den vergangenen Jahrzehnten in allen entsprechenden internationalen Standards in seiner Bedeutung hervorgehoben, und es wurden besondere Mechanismen und Instanzen zur Beobachtung der Einhaltung dieses wichtigen Prinzips eingerichtet. Hierzu dient unter anderem das Zusatzprotokoll (Fakultativprotokoll) zur UN-Deklaration gegen Folter (Optional Protocol to the Convention against Torture and other Cruel, Inhuman or Degrading Treatment or Punishment, OPCAT), das u.a. die Installierung unabhängiger Institutionen in den Signatarstaaten als nationale Mechanismen (zum Beispiel im Rahmen von Ombuds-Institutionen oder Volksanwaltschaften) und den Zugang zu Haftorten für ein zu etablierendes UN-Komitee (Subcommittee on Prevention of Torture; SPT) verpflichtend vorsieht. ${ }^{7}$

Das Zusatzprotokoll wurde allerdings erst von einem kleineren Prozentsatz der UN-Staaten - und dies teilweise auch mit Vorbehalten - unterzeichnet. Mit 2019 haben 100 Staaten das Zusatzprotokoll unterfertigt, das erstmals ein effektives Monitoring ermöglicht und die angeführten konkreten Schutzmechanismen in der Umsetzung der UN-Konvention vorsieht. Zusätzlich wurden auf internationaler Ebene das UN-AntiFolterkomitee sowie das Amt des UN-Sonderberichterstatters zu Folter, das über viele Jahre von einem Österreicher, Professor Manfred Nowak, ausgeübt wurde, eingerichtet.

Mit der Stärkung der regionalen Vernetzungen wurde aufgrund der Bedeutung des absoluten Folterverbots auch von der EU eine Antifolterkommission zur Unterstützung der Umsetzung eingerichtet (SoRENSEN 1991), die allerdings im Vergleich zur UN-AntiFolterkommission einem erweiterten Mandat, das die Überprüfung jeder Form der Freiheitsbeschränkung beinhaltet, zu folgen hat. Sie überprüft beispielsweise daher auch psychiatrische Krankenhäuser oder Altersheime und ähnliche Einrichtungen, in denen ein Missbrauchsrisiko besteht.

Ein weiteres Instrument in der Umsetzung der Anti-Folterkonvention sind forensische (gerichtsmedizinische) Standards, nämlich das Minnesota- (ToPraK 2009) und das Istanbul-Protokoll, auf die an späterer Stelle näher eingegangen werden soll. Sie sind von besonderer Relevanz für die Schnittstelle zwischen Medizin, Psychologie, Ethnologie und Rechtswissenschaften.

\footnotetext{
6 Vgl. $<$ https://www.ohchr.org/EN/ProfessionalInterest/Pages/CAT.aspx $>$ [Zugriff: 21-012020].

7 Vgl. $\quad<$ https://www.ohchr.org/Documents/Publications/training8Revlen.pdf $>$, Chapter 1; [Zugriff: $21-01-2020]$.
} 


\section{Zur Verbreitung von Folter}

Trotz des eingangs beschriebenen, eindeutigen Verbots und der komplexen Schutzmechanismen sind die Anwendung von Folter sowie unmenschliche und erniedrigende Behandlung wie auch ähnlich schwere Menschenrechtsverletzungen international leider eher im Zu- als im Abnehmen begriffen (Busch et al. 2015; WenZEL, KienZLER et al. 2015). Internationale Zahlen können selbstverständlich kein endgültiges und exaktes Bild der Häufigkeit und der Formen von Folter bieten, belegen aber deren breite Anwendung. So schätzt Amnesty International, dass in 141 Staaten Folter angewandt wird. ${ }^{8}$

In internationalen Statistiken wird dabei häufig zwischen der systematischen und der nicht systematischen, d.h. der nur zeitweise oder lokalisiert auftretenden, Anwendung von Folter unterschieden.

\subsection{Verschwunden, ermordet}

Man sollte nicht aus den Augen verlieren, dass auch das „Verschwindenlassen“ bzw. die illegale Ermordung oder Hinrichtung oft Teil oder Folge von Folter sind, aber auch unabhängig von dieser als eine schwere Menschenrechtsverletzung in Ländern, in denen auch gefoltert wird, angewandt wird. Das Schicksal solcher verschwundener „Disparechidos“ (SмIтH 2017) in Argentinien und anderen lateinamerikanischen Ländern, die im Falle von Argentinien nach Folter von Hubschraubern aus ins Meer geworfen worden waren (EsPARZA et al. 2010; VERBITSKY 1996), ist bis heute ein Problem für die Rehabilitation und Heilung von Angehörigen wie auch der demokratischen Zivilgesellschaft selbst in dieser und anderen Regionen (Sмітн 2017), auf das weiter unten noch einzugehen ist. In Argentinien beschäftigt zusätzlich das Problem der während der Regimezeit entführten Kinder Gerichte und Angehörige (CHELALA 1997). Die Bedeutung der psychologischen und emotionalen Belastung von Angehörigen durch das Fehlen einer adäquaten Aufklärung des Schicksals von Verschwundenen wurde auch vor kurzem vom Europäischen Gerichtshof für Menschenrechte anerkannt, der den Angehörigen der während der türkischen Invasion von Zypern (Operation „Attilas“) „,verschwundenen“ (und wahrscheinlich ermordeten) Zypriot/inn/en eine hohe Entschädigung zugesprochen hat. Die Begründung betont die Aufgabe des Staates, in diesem Fall der Türkei, die während der Invasion die vollständige Kontrolle des Landes innehatte, eine rasche und effektive Untersuchung des Schicksals der Betroffenen einzuleiten, was aber seitens der Türkei bis heute verabsäumt wurde. ${ }^{9}$ Die finanzielle Entschädigung von Angehörigen kann dabei selbstverständlich nicht den verlorenen Angehörigen ersetzen, allerdings ist es wichtig, Menschenrechtsverpflichtungen besonders auch von Staaten einzufordern und klare Zeichen bezüglich der Unrechtmäßigkeit bestimmter Handlun-

$8 \quad$ Vgl. <https://www.amnesty.org/en/get-involved/stop-torture/> [Zugriff: 21-01-2020].

9 Vgl. Application no. 25781/94, judgement 2001, siehe $<$ https://hudoc.echr.coe.int $>$ [Zugriff: 22-01-2020]. 
gen zu setzen. Des Weiteren soll die Position von Opfern und Angehörigen vermehrt gestärkt werden.

\subsection{Folter als Teilfaktor von Verfolgungshandlungen}

Folter ist auch oft Teil von Verfolgungshandlungen oder sogar Genozid und Bürgerkriegshandlungen und somit als traumatisches Ereignis für das Individuum, Familien sowie ethnisch oder politisch definierte Gruppen eingebettet in weitere äußerst belastende Erfahrungen (Wenzel 2007). Bei einigen ethnischen Gruppen, beispielsweise bei Armenier/inn/en, Kurd/inn/en oder Yezid/inn/en ist dies auch nicht auf einmalige schwerwiegende Ereignisse beschränkt, sondern Teil einer oft über Generationen anhaltenden Strategie von Verfolgung und systematischen Auslöschungsversuchen. Man könnte hier von einer genozidalen Umgebung (,genocidal environment“) sprechen. $\mathrm{Zu}$ den psychosozialen Folgen dieser auch Generationen überschreitenden Interaktion mit einer nicht nur vorübergehend, sondern langfristig anhaltend, zerstörenden sozialen und politischen Umwelt liegen bisher noch kaum Studien vor, allerdings könnte man viele Forschungsergebnisse, die sich auf jüdische oder armenische Gruppen beziehen, in diesem Kontext neu interpretieren (ANTON et al. 2011; BoHLEBER 2007; WeInGARTEN 2004; Sorscher \& CoHEN 1997; Adelman 1995). Zu berücksichtigen sind in der Weitergabe von Traumata neben der Eltern-Kind-Interaktion und dem sozialen Umfeld wahrscheinlich auch epigenetische Aspekte (JAwaID et al. 2018; Kellermann 2013). Für die meisten dieser Gruppen besteht kaum die Möglichkeit einer Erholung oder der Erfahrung von Sicherheit, was auch den Aufbau besonderer Bewältigungsstrategien voraussetzt, die bis auf einige wenige Studien vor allem bei jüdischen, von der Shoah betroffenen Gruppen (Kellermann 2001; Kirmayer et al. 2014; Shrira et al. 2011) bislang ebenfalls kaum erforscht ist. Es kann davon ausgegangen werden, dass die politischen Strategien der Verfolger darauf abzielen, beispielsweise durch die Vernichtung von kultureller Identität (KINGSTON 2015) im Fall der Türkei gegen Kurd/inn/en und Armenier/innen, diese Bewältigungsstrategien zu stören oder zu verhindern. Folter kann hier als Teilstrategie dienen, mögliche Resilienz und den Widerstand stützende Mitglieder der Gemeinschaft psychologisch zu brechen oder zu neutralisieren.

\subsection{Voraussetzungen der Folter und schwerwiegender Menschen- rechtsverletzungen - individual- und gruppendynamische Aspekte}

Die bereits erwähnte, eher ansteigende Häufigkeit extremer Gewalt wie Folter trotz der Entwicklung und zunehmenden Ausbreitung des Konzepts der Menschenrechte hat immer wieder zur Frage veranlasst, wer die Täter/innen solcher offenbar die grundlegende Menschlichkeit verletzenden Handlungen sind oder auch wie Gruppen oft schein- 
bar über Nacht, wie im Fall von Ruanda oder Bosnien, zu Massenmörder/inne/n werden können (DUDAI 2006). Eines der besonders erschreckenden Ergebnisse der Forschung zu dieser Fragestellung seit dem Zweiten Weltkrieg, die allerdings in Folge immer wieder reproduziert wurden, ist die Beobachtung, dass es sich bei den Täter/inne/n weder um psychisch kranke Ausnahmepersönlichkeiten noch um auffällige, gewalttätige oder offensichtliche „Monster“ handelt (DUDAI 2006). Während die Serienmörder/innen der Kriminalstatistik sehr häufig durch Gewaltakte beispielsweise gegenüber Tieren bereits in der Kindheit auffällig werden (ARLuKE et al. 2018), sind Täter/innen extremer politischer Gewalt oft scheinbar perfekt angepasste, unauffällige, gute Staatsbürger/innen und Nachbar/inne/n, sodass einige der ersten dazu publizierenden Expert/inn/en wie Hannah Arendt und Arno Grün von der Banalität des Bösen oder vom „Wahnsinn der Normalität“ (Gruen 1992; HaSlam \& ReICHER 2007) sprachen. Der griechische Forscher Haritas Fatouros, der eine der ersten Untersuchungen zur Entstehung der Voraussetzungen von Folter während der griechischen Diktatur durchgeführt und in der Folge publiziert hat (HARITOS-FATOuRos 1988), betonte die Bedeutung von Indoktrination und selbst erlittener Folter oder Gewalt in der Ausbildung von Folterern im Rahmen der Militärausbildung bei zuvor unauffälligen jungen Männern vor allem aus ländlichen Gemeinden. Ähnliche Mechanismen können auch bei der Ausbildung von Kindersoldat/inn/en, beispielsweise im Kongo und in Uganda (BETANCOURT 2008; KLASEN et al. 2011), beobachtet werden. Die Tatsache, dass einige der Täter/innen zuvor selber Opfer waren, soll allerdings nicht von Schuld und Verantwortung der Täter/innen ablenken, sondern nur die Bedeutung der Gesellschaft in langfristigen Präventions- und allenfalls späteren Versöhnungsprozessen betonen (BRACKEN et al. 1996; CoRBIN 2008). Folgt man publizierten Täter/innenprofilen, so wird dabei auch deutlich, dass neben dieser Gruppe der Täter/innen auch persönlichkeitsgestörte Individuen mit erhöhter Gewaltbereitschaft und geringer Empathie und ideologische Überzeugungstäter/innen (GRIFFIN 2017) zum Täter/innenkreis gehören, der sich in der Beschreibung nicht für jeden Fall durch ein einzelnes universelles Modell oder Modelle wie Liftons „doubling“ (GRIFFIN 2017), das erfassen lässt (siehe auch die Liste der beobachteten Gründe und Rechtfertigungen für Folter oben). Lifton beschrieb eine Quasi-Spaltung des Selbst, die es ermöglicht, mit dem Gewissen nicht vereinbare Handlungen ausführen zu können und dem vorhandenen inneren „,bösen“ Anteil ohne Selbstreflektion Raum zu geben.

SAPOLSKY und andere Humanethologen haben auf die biologischen Aspekte von Aggressionshemmung, Empathie und Aggressionsauslösern hingewiesen (SAPOLSKY 2017). Wie auch bei Gruppen können in der Ausbildung von Folterern neben selbst erlittener Gewalt, auch Drohungen oder allgemeine Sozialisationsmechanismen beobachtet werden (KLASEN et al. 2015; PHAm et al. 2009), die auch bei Gewalt von Gruppen gegenüber anderen Gruppen im Rahmen von Massakern und Genozid in der Vorbereitung dokumentiert sind. Dies betrifft beispielsweise im Falle von Ruanda die Abfolge von Manipulationen, die im vorbildlichen Genozidmuseum ${ }^{10}$ in Kigali Schulkindern, aber auch Besucher/inne/n aus der ganzen Welt nahegebracht werden, was die Bedeu-

10 Vgl. <https://www.kgm.rw/> [Zugriff: 22-01-2020]. 
tung der Verflechtung der Erforschung und der Prävention von Bereitschaft zu extremer Gewalt unterstreicht. In Ruanda erfolgte eine langsame, aber intensive und nachhaltige Manipulation des Bildes von Nachbar/inne/n über das Radio, das auch in Dörfern ohne Stromversorgung über Batterien betrieben und somit allen Haushalten zur Verfügung stand. Die Manipulation der Repräsentation „des Anderen“ erfolgte dabei durch auch in anderen Situationen zu beobachtende Schritte der symbolischen Entmenschlichung, also des Abbaus von Tötungshemmung durch Manipulation, nämlich:

- Der/die „Andere“ ist anders.

- Er/sie ist nicht gleichwertig.

- Er/sie ist Teil einer (gefährlichen) Gruppe.

- Er/sie ist kein Mensch, sondern (wie) ein Tier.

- Er/sie ist Ungeziefer oder Unkraut.

- Das daher nicht nur beseitigt werden darf, sondern muss.

Auch komplexe Symbole der Gruppe, wie beispielsweise die der gewählten („,chosen“) bzw. konstruierten Traumata, die erstmals von VoLKAN (1997) beschrieben wurden, können offenbar der Vorbereitung von Gewalt besonders im ethnischen und interkulturellen Kontext dienen. Bei der Entstehung dieser „konstruierten“ Traumata werden in der Regel länger zurückliegende Ereignisse, meist solche mit negativer Bedeutung, wie militärische Niederlagen, zu einem wesentlichen, die Gruppe definierenden gemeinsamen Symbol „ernannt“, um das sich die Gruppenidentität konstituieren lässt (VoLKAN 1997; 2006). Ein klassisches Beispiel hierfür ist die Verbreitung des Motivs des Amselfeldes (Kosovo Polje) und der (angeblich) dramatisch verlorenen Schlacht des serbischen „Heiligen Zar Lazar“ im Kampf zwischen serbischem und türkischem Heer. Die objektive Geschichtsforschung der vergangenen Jahrzehnte hat gezeigt, dass die meisten Aspekte des um dieses Ereignis aufgebauten Mythos eine deutliche Verzerrung der historischen Realität darstellen. VoLKan hat darauf hingewiesen, dass die im Rahmen der Implementierung eines solchen - mehr symbolisch konstruierten als realen - Traumas geförderten Gruppenprozesse eine massive Gruppenregression einleiten können, die dazu führt, dass die Fähigkeit zur Realitätsprüfung und sachlichen Wertung von Ereignissen bei den Mitgliedern der Gruppe beeinträchtigt wird. Diese werden damit empfänglich für Manipulation durch falsche Führer („,bad leaders“) (VOLKAN 2004). Es entsteht eine Situation, wie im Fall von Ruanda oder bei den Massakern im ehemaligen Jugoslawien, in der sie davon überzeugt werden können, eigentlich vertraue Nachbar/inne/n, mit denen man kurz zuvor noch gefeiert hat, als fremde und gefährliche Gruppe („die Anderen“) zu sehen und abzuwerten oder als Bedrohung zu definieren. Dies ermöglicht es später, auch extreme Gewalt zu rechtfertigen oder sogar zu fordern. Denkmäler oder symbolische Objekte, im Falle der Serb/inn/en im Kosovo die Pfingst- 
rose und die Denkmäler des Heiligen Lazar sowie der Schlacht auf dem Amselfeld, unterstützen diesen Prozess in der Propaganda, um Emotionen gleichsam „bündeln“ zu können.

\subsection{Folgen der Folter}

Die schweren Langzeitfolgen von Folter sind wohl eines der wichtigsten Argumente gegen ihre Anwendung. Dabei ist zu berücksichtigen, dass physische und psychologische Folter in der Praxis nicht wirklich zu trennen sind. Physischer Schmerz führt unmittelbar und auch bei chronischen Schmerzerkrankungen, die für Folter charakteristisch und äußerst schwer zu behandeln sind, zu neurobiologischen Veränderungen. Körperliche Folgen wie schwere Verletzungen, bei Folter oft der Gelenke, erinnern immer wieder an die erlittene Folter und fördern somit ebenso wie Umgebungssituationen, die an Folter erinnern (situative ,Trigger"), das Wiederaufleben traumatischer Erinnerungen. Psychologische Folter ist immer auch von vorübergehenden oder langfristig chronischen Folgen im Bereich des Gehirns und der Transmitter (ZANDIEH et al. 2016) (Botenüberträgersysteme des Gehirns) und möglicherweise sogar von epigenetischen Veränderungen (ALmLi et al. 2014) begleitet. Der Einfluss psychologischer Faktoren auf die Allgemeingesundheit wurde dabei lange unterschätzt. So geht man heute davon aus, dass z.B. chronische Schlafstörungen, unter denen fast jede/r Folterüberlebende leidet, zu einer auch körperlich bedingten Verringerung der Lebenserwartung und erheblicher Beeinträchtigung im Alltag führen (AsPLUND 1999). Ein weiterer unterschätzter Faktor sind die Langzeitfolgen der ebenfalls bei fast allen Folterüberlebenden zu beobachtenden stumpfen Schädel-Hirn-Traumata durch Schläge, Stöße oder Stürze, die ebenfalls zu medizinisch schwer zu behandelnden Beeinträchtigungen wie Konzentrationsstörungen, Kopfschmerzen und verringerter Stresstoleranz führen können (MorENo \& GRODIN 2002; MAC Donald et al. 2001; FernandeZ et al. 2001).

Historisch ist zu berücksichtigen, dass die heute sehr weit entwickelte Traumafolgeforschung über lange Zeit kaum verbindliche Modelle für psychologische Traumatisierung zur Verfügung stellte, lange wurde auch der Fokus hauptsächlich auf Traumafolgestörungen bei Soldaten gelegt, beispielsweise auf die „Kriegzitterer“ des Ersten Weltkrieges (PECKL 2007), während z.B. Kriegstraumafolgen bei Zivilist/inn/en zu wenig berücksichtigt wurden. Schwerpunkt war hier auch nicht immer die Heilung oder vollständige Rehabilitation, sondern die Wiederherstellung der Kriegstauglichkeit. Dieses Problem ist bis heute in der Begutachtung bestimmter traumatisierter Gruppen, z.B. von Opfern von Gewalt in Kinderheimen in Deutschland und Österreich, die den aktuellen Wissenstand ignoriert, zu beobachten. Erst mit den Studien über Shoah- und Konzentrationslagerüberlebende von Frankl, Niederland und anderen erfolgte eine ernsthafte Auseinandersetzung, in der Anfangsphase noch mit einem Fokus auf körperliche und hirnorganische Folgen von Unterernährung oder Kopfverletzungen. Schließlich wurde das (Konzentrationslager-)Überlebendensyndrom (Bower 1994; Peters 
1989) zunehmend als solches anerkannt. Die Entwicklung des Konzepts der heute als besonders charakteristisch gesehenen posttraumatischen Belastungsstörung (PTSD/ PTBS) begann ebenfalls mit einer Initiative zur Unterstützung von (US-) Kriegsveteranen des Vietnamkriegs, denen aufgrund einer fehlenden adäquaten Diagnosekategorie Unterstützung und medizinisch-psychologische Versorgung vorenthalten wurden. Da deutlich wurde, dass das ursprünglich sehr einfache Diagnosemodell der PTSD nicht die Komplexität psychologischer Traumafolgen widerspiegeln kann, wurde das Konzept zunehmend erweitert (NORTH et al. 2016). Deutlich wurde auch, dass neben den Symptomen der PTBS wie quälenden Erinnerungen an die erlittenen Erfahrungen während des Tages und in Form von Albträumen, verstärkter Ängstlichkeit und anderen Stressreaktionen, Vermeidung von Erinnerungen, Konzentrationsstörungen oder dissoziativen Symptomen mit momentaner Abwesenheit auch die häufigen Begleiterkrankungen sowohl bei Soldaten wie auch bei Folterüberlebenden zu berücksichtigen sind. Dazu gehören unter anderem schwere Depressionen (WENzEL et al. 2000), chronische therapieresistente Schmerzen, ein erhöhtes Suizidrisiko (WENZEL et al. 2009), reaktive Psychosen (Wenzel et al. 1999), die Selbstmedikation mit Alkohol, Benzodiazepinen oder Drogen, sexuelle Probleme und Konflikte mit Partner/in und Familie. Viele Betroffene vermeiden eine Behandlung aufgrund von Scham, posttraumatischem Vermeidungsverhalten, fehlender finanzieller Mittel oder wegen der Stigmatisierung psychologischer Symptome, die in vielen Ländern zu beobachten ist. Es ist daher wichtig, den Betroffenen, ihren Angehörigen und der Gesellschaft deutlich zu machen, dass psychologische Folgen nach extremen Erfahrungen eine normale Reaktion auf eine abnorme Situation darstellen und weder als Schwäche noch als Geisteskrankheit zu interpretieren sind.

Neben den hinsichtlich der neurobiologischen Grundmechanismen primär kulturunabhängigen (wenn auch kulturüberlagerten) und auch humanethologisch als adaptiv zu interpretierenden Folgeerkrankungen wie der PTBS wurden in den letzten Jahren auch in der aktuellen Fassung des Standardhandbuchs psychiatrischer Diagnosen (DSM 5) zunehmend kulturelle Aspekte von Krankheitsverhalten und Krankheitsverarbeitung sowie kulturspezifische Reaktionsformen auf extreme Belastungen (,,idioms of distress“) berücksichtigt (WenZel, Frewer et al. 2015). So können quälende Besuche unzufriedener Geister von Ermordeten im Traum oder während des Tages erlebt werden (ToBIN \& FrIEDMAN 1983). Allerdings liegen derzeit nur Daten zu traumaspezifischen Reaktionsformen in einigen Kulturen vor, ein universelles Handbuch oder eine entsprechende Datenbank sind als Wunschprojekt der Zukunft und möglicherweise unrealistische Forderung zu sehen. 


\section{Aspekte der Behandlung}

Die Betreuung von Überlebenden wird auch dadurch erschwert, dass viele Ärzt/ inn/e/n in jenen Ländern, in denen die Folter erfolgte, Angst haben Opfer zu behandeln, da sie unter Umständen selber mit Verfolgung oder Sanktionen oder sogar Ermordung rechnen müssen. Auch haben viele Opfer aus finanziellen und geographischen Gründen in diesen Staaten keinen Zugang zu Behandlung, obwohl - oft mit internationaler Unterstützung - spezielle Behandlungszentren für Folteropfer, allerdings meist nur in Großstädten, eingerichtet wurden. ${ }^{11}$

\subsection{Angehörige und Familien als indirekte Folteropfer}

Die Betreuung von Angehörigen, besonders während der Haft und Folter, wenn das Schicksal der Familienmitglieder oft noch unklar ist, oder im Fall ihrer Ermordung, ist wie bereits angemerkt, ein ebenfalls noch zu wenig untersuchtes und gelöstes Problem. Angehörige werden oft bedroht oder stigmatisiert, wobei sich die gegenseitige Solidarisierung, dort wo sie ohne zu große Gefahr möglich ist, sich als die vorläufig beste Lösung erwiesen hat. Als Beispiel können hier die „Madres de la Plaza Major“ während der Zeit der argentinischen Junta-Diktatur dienen (REInEman 2011). Auch nach der Rückkehr von Überlebenden zu ihren Familien ist besondere Unterstützung nötig, da Trauma-Folgeerkrankungen der Folterüberlebenden unter Umständen mit für die Familien schwer zu lösenden Symptomen wie vermehrter Irritierbarkeit oder Alkoholmissbrauch als Selbstbehandlung einhergehen können. Selbst Psychiater/innen und Psychotherapeut/inn/en, die Opfer behandeln, benötigen besondere Unterstützung, um nicht selbst im Rahmen der Behandlung sekundär traumatisiert zu werden oder ein Burnout zu erleiden (IzGuiERDo et al. 2019).

\subsection{Weitere Aspekte der Behandlung}

Die Behandlung von Überlebenden muss dabei trauma- und kultursensitiv erfolgen, was besondere Anforderungen an Ärzt/inn/e/n und Therapeut/inn/en stellt, da dieser Aspekt auch in der klassischen Psychotherapieausbildung oft kaum berücksichtigt wird (Wenzel, Drozdek et al. 2019). Neue Therapietechniken wie Eye Movement Desensitization and Reprocessing (EMDR) (die Reduktion intrusiver Erinnerungen durch Rekonfrontation mit diesen in der Vorstellung, kombiniert mit Blickfolgebewegungen) und Behandlungsformen wie narrative Expositionstherapie (NET) (VILLER et al. 2017; Adenauer et al. 2011; Hensel-Dittmann et al. 2011; Halvorsen 2010) werden international in Kriegsgebieten, aber auch mit geflüchteten Überlebenden zunehmend

$11 \quad$ Vgl. <www.irct.org > [Zugriff: 20-01-2020]. 
weiterentwickelt und in der Behandlung eingesetzt. Einige Therapeut/inn/en versuchen dabei etablierte Traumatherapieformen wie die während der Juntazeit in Lateinamerika entwickelte „Testimony“-Therapie, bei der in der Therapie Zeugnis gegen Täter/ innen abgelegt wird, mit kultursensitiven Modellen wie Ritualen zu verbinden (EsALA \& TAING 2017) oder Gruppen- und Einzeltherapien - ebenfalls kultursensitiv - zu integrieren (FERNANDES \& AiELlo 2018).

In den vergangenen Jahrzehnten wurde zusätzlich deutlich, dass ein interdisziplinärer Gruppen- und sozialorientierter („community oriented“) Ansatz gegenüber der reinen Behandlung von Einzelpersonen von wesentlicher Bedeutung ist. Seitens internationaler Organisationen wurde der Ansatz der MHPSS (Mental Health and Psychosocial Services) (ADAKU et al. 2016; ToL et al. 2015) entwickelt, der besonders Gruppen und Gemeinschaften als Handlungsträger der Rehabilitation als Alternative zur individuellen Behandlung durch Spezialist/inn/en wie etwa Psychiater/inne/n in den Vordergrund stellt. In Ghaza wurde deshalb beispielsweise das Ghaza Community Mental Health Project eingerichtet, ${ }^{12}$ das besonders auch Folteropfern einfachen und gemeindenahen Zugang zu Behandlung ermöglichen soll. Derzeit liegen aus wissenschaftlicher Sicht allerdings noch nicht genügend Daten vor, um die Vorteile beider Modelle in der Praxis und in der „universellen Anwendung“ valide einschätzen zu können (WENZEL, VöLKL-KeRnstock et al. 2019). Sicher ist jedenfalls, dass in den Herkunftsländern vieler Opfer, in denen Folter endemisch ist, die üblichen Versorgungssysteme meist bereits mit den alltäglichen Problemen außerhalb von Gewaltfolgen überlastet sowie qualitativ und quantitativ unzureichend und überdies meist nicht leistbar für Betroffene sind. Auch sind Behandlungsmodelle aus ökonomisch reicheren Ländern meist nicht oder nur begrenzt anwendbar (Wenzel et al. 2015). Es ist daher von entscheidender Bedeutung, in der Versorgung von Kriegs- und Folteropfern effektive und praktisch umsetzbare Strategien wie MHPSS zu entwickeln, zu evaluieren und auch international zu fördern.

\subsection{Transitional Justice - Therapeutic Justice}

Ein besonders wichtiger Aspekt dieser Ausweitung von Interventionsansätzen ist der Umgang mit der Situation in Ländern, in denen Gewalt und Folter so häufig sind, dass sie über lange Zeit praktisch zum Alltag gehört haben. Nach Restabilisierung der politischen, sozialen und juristischen Situation ist es oft nötig, Lösungen für Situationen zu finden, in denen große Teile der Bevölkerung oder politischer Parteien als Täter in Menschenrechtsverletzungen direkt oder indirekt involviert waren. Diesem Ziel dienen beispielsweise Wahrheits- und Wiederannäherungskommissionen (,truth and reconciliation committees“) (WILSON 2000; STEIN 1998; WELSH 1998), beispielsweise in Südafrika, oder in anderen Ländern traditionelle Gerichtshöfe wie die Gacaca-Gerichtshöfe in Ruanda (RutaYISIRE 2014, p. 74). Die bisherigen, in noch zu geringem Umfang vorliegenden wissenschaftlichen Studien zur Effizienz dieser Modelle vermitteln ein wider-

$12 \quad$ Vgl. <https://gcmhp.ps> [Zugriff: 22-01-2020]. 
sprüchliches Bild (RUTAYISIRE 2014) und machen deutlich, dass eine sorgfältige und kultursensitive Planung und nachhaltige Umsetzung entscheidend für ihren Einsatz sind.

\subsection{Zur Schnittstelle Medizin, Psychologie und Recht}

Besonders im Bereich schwerwiegender Menschenrechtsverletzungen wie der Folter ist eine interdisziplinäre Zusammenarbeit zwischen Gesundheitsberufen, Jurist/ inn/en und Rechtssystem oft von entscheidender Bedeutung. Das betrifft sowohl die Prävention wie auch den Schutz und die Unterstützung von Betroffenen, einschließlich von Überlebenden und Angehörigen, und schließlich den Schutz von rechtsstaatlichen, demokratischen und zivilgesellschaftlichen Strukturen in der Gesellschaft. Dafür ist es wichtig, dass Folter und Folterfolgen erkannt, dokumentiert und gerichtlich oder durch unabhängige Kommissionen oder sogar internationale Gerichtshöfe nachhaltig rasch und effizient der Strafverfolgung zugeführt werden.

\subsection{Die Rolle von Gesundheitsberufen im Bereich des Menschenrechtsschutzes und der Folterprävention}

Ärzt/inn/e/n, Psycholog/inn/en und andere Gesundheitsberufe spielen im Kampf gegen Folter eine wichtige Rolle, da körperliche und psychologische Folgen wichtige Beweismittel und oft erste Auslöser einer entsprechenden rechtlichen Untersuchung sind. Dabei sind vor allem zwei Probleme zu berücksichtigen: Erstens besteht zumindest in dem Land, in dem Folter angewandt wird oder keine ausreichenden rechtsstaatlichen Strukturen bestehen, oft eine unmittelbare Gefahr für die Angehörigen von Gesundheitsberufen, die Folter erkennen, behandeln, dokumentieren oder sie zu verhindern versuchen und somit ihren ethischen Verpflichtungen nachkommen. In aktuellen Situationen wie beispielsweise bei der willkürlichen Verhaftung von Ärzt/inn/en vor allem im Iran und in der Türkei hat der Weltärzteverband jeweils sehr rasch und nachhaltig reagiert (Wenzel, den Otter et al. 2019; Mirzaei 2019; Perez-Sales 2018), um Einspruch gegen die Verfolgung von vor allem Ärzt/inn/en geltend zu machen. Auch die Vereinten Nationen haben sich nachdrücklich für die Unterstützung von „human rights defenders“ ${ }^{13}$ also im Menschenrechtsschutz aktiven Zivilist/inn/en eingesetzt, allerdings sind die bisherigen Maßnahmen sicher unzureichend, um einen nachhaltigen Schutz zu gewährleisten. Zweitens sind die Ausbildungscurricula der meisten Gesundheitsberufe unzureichend, um zu lernen, forensische Fragestellungen abzudecken, also Gewaltfolgen gerichtsmedizinisch und gerichtlich verwertbar zu dokumentieren und entsprechend brauchbare Befunde zu erstellen. Entsprechende Standards und Trainingsmaßnahmen sind daher von wesentlicher Bedeutung. Dem dienen, wie bereits angemerkt, vor allem das Minnesota-Protokoll, das der korrekten Untersuchung von Ermordeten dient, und

13 Siehe z.B. Deklaration 72/247 vom 24. Dezember 2017. 
das Istanbul-Protokoll, das als Reaktion auf einen Folterfall in der Türkei entwickelt wurde und weiter unten ausführlicher behandelt wird.

Es ist leider auch Teil der Realität, dass Ärzt/inn/e/n und Angehörige von anderen Gesundheitsberufen oft bereit sind, sich an Folter direkt oder indirekt zu beteiligen oder deren Anwendung, auch ohne drohende Gefahr für ihre eigene Sicherheit, zu verschleiern. Dies wird am Beispiel der amerikanischen Psycholog/inn/en, denen die Mitarbeit an der Entwicklung von Foltertechniken und deren Verteidigung vorgeworfen wird, deutlich (DYER 2016). Im Gegensatz dazu haben die meisten nationalen und vor allem internationalen Berufsdachorganisationen eindeutig klargestellt, dass jede Unterstützung oder Mitarbeit bei Folter durch ihre Berufsmitglieder eine schwere Verletzung der Berufsethik darstellt. Insbesondere der Weltärzteverband sowie der Weltpsychiatrieverband haben diesbezüglich immer wieder eindeutig Stellung bezogen (Perez-SALES et al. 2018; Wilson 1983; HelmCHen \& OKASHa 2000), während der amerikanische Psychologenverband erst sehr verzögert und nach heftiger internationaler Kritik reagierte (BoHANnOn 2015; Boyd et al. 2014; Olson et al. 2008).

Ein Vorteil von entsprechenden Untersuchungen und Dokumentationstandards ist es in diesem Zusammenhang, dass es für Ärzt/inn/e/n, die aus Angst oder Gefälligkeit für ein Regime Falschgutachten zur Verschleierung von Folter und Menschenrechtsverletzungen erstellen, schwieriger ist, Folter zu ignorieren, zu entschuldigen oder auch einfach nicht zu dokumentieren. Wir wollen in Folge daher kurz auf das Istanbul-Protokoll eingehen, das als gutes Beispiel für ein Werkzeug im Kampf gegen Folter zu sehen ist.

\section{Das Istanbul-Protokoll}

Das Istanbul-Protokoll (Manual on Effective Investigation and Documentation of Torture and Other Cruel, Inhuman or Degrading Treatment or Punishment, 1999) wurde, wie bereits angemerkt, als Reaktion auf einen konkreten Foltervorfall in der Türkei entwickelt, bei dem das inzwischen erweiterte und ergänzte Minnesota-Protokoll Anwendung fand (Wenzel et al. 2012). Die Angehörigen eines angeblich an einer alltäglichen Erkrankung verstorbenen Häftlings hatten dabei bei der Waschung und Vorbereitung für die Beerdigung des Opfers offensichtliche Gewaltfolgen, die in klarem Widerspruch zu den Angaben des Gefängnisarztes standen, festgestellt und fotografisch dokumentiert, sodass eine Strafverfolgung durchgeführt werden konnte. Eine internationale, interdisziplinäre Expert/inn/engruppe stellte es sich daher zur Aufgabe, einen dem MinnesotaProtokoll vergleichbaren Standard für die Untersuchung von überlebenden Opfern zu entwickeln (FrEwer et al. 2012). Dieses aufgrund des interdisziplinären Ansatzes sehr umfangreiche Dokument ${ }^{14}$ wurde schließlich von der Generalversammlung der Verein14 Deutsche Übersetzung: <https://www.vr-elibrary.de/isbn/9783847100300> [Zugriff: 20-022020]. 
ten Nationen als Standard für die Untersuchung von Foltervorwürfen angenommen und in die Reihe der Trainingshandbücher des UN-Hochkommissars für Menschenrechte integriert sowie in eine Reihe von Sprachen übersetzt. Verschiedene regionale und internationale Organisationen, besonders die EU, haben die Verbreitung und Anwendung des Protokolls in Folge gefördert, hierzu dienten Projekte wie das Istanbul Protocol Implementation Project (IPIP) des International rehabilitation Councils for Torture Survivors (IRCT) und das von Österreich aus koordinierte ARTIP (Awareness Raising and Training for the Istanbul Project) EU Projekt. ${ }^{15}$ Internationale Gerichtshöfe sowie Einrichtungen wie das Anti- Folter Komitee der Vereinten Nationen oder der EU sind auf zuverlässige Befunde, die als Beweismittel eingesetzt werden können, angewiesen und unterstützen somit die Anwendung des Protokolls ebenfalls. Das Istanbul Protokoll besteht aus sechs Kapiteln sowie mehreren begleitenden Anhängen (WENZEL et al. 2012, p. 1): Relevante internationale rechtliche Standards, 2) Relevante ethische Kodizes, 3) Rechtliche Untersuchung von Folter, 4) Allgemeine Hinweise für Befragungen, 5) Der Nachweis von physischen Folgen der Folter, 6) Der Nachweis von psychischen Folgen der Folter.

Trotz der Stigmatisierung von psychologischen Folgen oder Symptomen sollte immer auch eine psychologische oder psychiatrische Untersuchung erfolgen, da einerseits psychische Folgen besonders häufig und langfristig sind, und die Tendenz, Folter so anzuwenden, dass physische Spuren nicht nachweisbar sind, Beweise in Form von psychologischen Folgen besonders gewichtig werden lässt. Schließlich können psychische Folterfolgen, aber auch andere, von Folter unabhängige psychische Probleme oder Erkrankungen, eine klare, eindeutige und widerspruchsfreie Aussage schwierig machen und die Glaubwürdigkeit des/r Überlebenden zu Unrecht in Frage stellen.

Das Protokoll betont dabei, ebenso wie die aktuellen EU-Richtlinien zur Berücksichtigung von Opfern im Strafverfahren (WENZEL, VöLKL-KERnSTOCK et al. 2019), den Schutz vor sekundärer Viktimisierung bzw. Retraumatisierung, also in diesem Zusammenhang die Notwendigkeit, medizinische und juristische Untersuchungen und Befragungen so durchzuführen, dass es nicht zu einer neuen oder erneuten Traumatisierung der Opfer, beispielsweise durch die Reaktivierung belastender Erinnerungen oder die psychologische Abwertung des Opfers, selbst kommt. Dies ist besonders für die Ausbildung nicht nur von Jurist/inn/en, sondern auch von Mediziner/inne/n und Psycholog/inn/en von besonderer Bedeutung, da dies sonst sogar von sonst gut ausgebildeten Expert/inn/en oft zu wenig berücksichtigt wird. Schließlich wird auch auf das Risiko einer sekundären Traumatisierung der eingebundenen Expert/inn/en selbst durch Konfrontation mit Folterfolgen hingewiesen.

Zu berücksichtigen ist, dass das Istanbul-Protokoll nicht als Handbuch allen medizinischen Wissens zu Folterfolgen zu sehen ist, da dieses aufgrund der raschen Entwicklung medizinischen Wissens fast wöchentlich zu aktualisieren wäre. Es handelt sich auch nicht um einen völkerrechtlichen Vertrag, der die Unterzeichnung oder Ratifizie-

$15 \quad$ Vgl. <http://www.istanbulprotocol.info/index.php/de/> [Zugriff: 20-02-2020]. 
rung der entsprechenden Staaten voraussetzt, sondern ist prinzipiell bei Foltervorwürfen anzuwenden. Das Protokoll enthält daher, wie in Abbildung 1 (siehe unten) ersichtlich, nicht nur Anleitungen zur medizinischen, psychologischen oder psychiatrischen Untersuchung, sondern auch Vorgaben zur Umsetzung einer unabhängigen Sachverhaltsanalyse durch entsprechende Kommissionen und Einrichtungen des Rechtsstaats und der Zivilgesellschaft und für Gefängnisbesuche. Bei diesen ist auch im Auge zu behalten, dass Gefängnisse und andere Haftanstalten, beispielsweise auch Schubhaftzentren, als besondere Risikosituationen hinsichtlich der Verletzung von Menschenrechten zu sehen sind. Hier sind neben dem Istanbul-Protokoll und der UN-Konvention gegen Folter auch weitere Menschenrechtsstandards wie die „Mandela Rules“ über Haftbedingungen allgemein ${ }^{16}$ oder die „Bangkok Rules“ zum Schutz von weiblichen Häftlingen ${ }^{17} \mathrm{zu}$ berücksichtigen.

\subsection{Das Istanbul-Protokoll im Asylverfahren}

Obwohl der Text des Istanbul-Protokolls in der derzeitigen Fassung besonders auf Situationen wie Gefängnisbesuche in den Ländern eingeht, in denen Foltervorwürfe unmittelbar untersucht werden sollen, ist es prinzipiell auf alle entsprechend relevanten Situationen anzuwenden. Dies gilt auch für die Aufnahmeländer im Asylverfahren (FrEWER et al. 2012). Hierbei ist zu berücksichtigen, dass die UN-Konvention gegen Folter von den entsprechenden Signatarstaaten der Konvention, zu denen auch beispielsweise Deutschland und Österreich gehören, den besonderen Schutz von Folteropfern auch gegen Rückschiebung (,refoulement“) in Art. 3 der Konvention vorsieht. Auch die EUAufnahmerichtlinien für Flüchtlinge definieren Folteropfer als vulnerable Gruppe, die einen besonderen Schutz genießen sollte (WEnzel, VöLKL-Kernstock et al. 2019).

Bei einem gut organisierten Aufnahmeverfahren sollte die entsprechende medizinische und psychologische Untersuchung besonders bei Verdacht oder Angaben zu Folterungen oder anderen schweren Gewalterfahrungen eine Reihe wichtiger Aufgaben (Abu SuHaiban et al. 2019) erfüllen (vgl. Abb.1).

Hierbei ist eine enge und gut organisierte Zusammenarbeit und die Einbindung entsprechender Ressourcen wesentlich, denn diese kann nicht von einer einzelnen Einrichtung, wie etwa den Asylämtern, geleistet werden. Sinnvoll wäre es nach unserer Erfahrung auch, wenn wesentliche Informationen, beispielsweise bezüglich der Hinweise auf Folterfolgen, bereits bei der Erstuntersuchung erhoben und dann Teil eines umfassenden Betreuungsplans würden, zu dem allenfalls auch eine Untersuchung nach dem Istanbul-Protokoll bei Foltervorwürfen und eine frühe medizinische und psychologische Intervention durch geeignete Spezialeinrichtungen gehören würden. Es ist auch

$16 \quad$ Vgl. $<$ https://www.penalreform.org/issues/prison-conditions/standard-minimum-rules/> [Zugriff: 22-02-2020].

17 Vgl. <https://www.penalreform.org/issues/women/bangkok-rules-2/> [Zugriff: 22-02-2020]. 
Abb. 1: Relevante Aspekte für die medizinische und psychologische Untersuchung bei Verdacht auf oder Angaben zu Folterungen in Drittländern

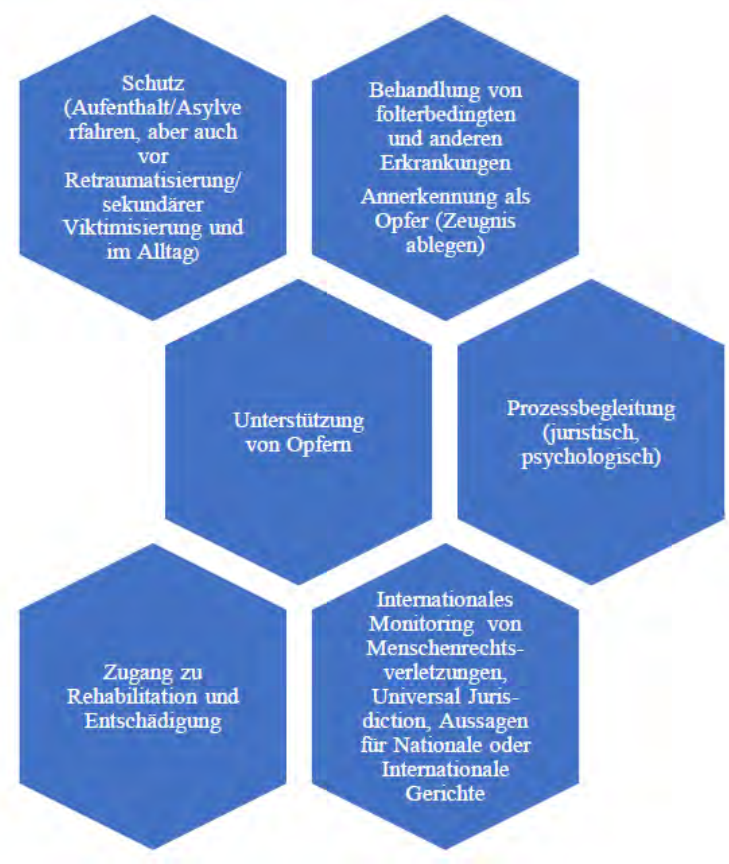

Quelle: Thomas Wenzel, Siroos MirzaeI, Reem AlksiRI: Training manual for ARTIP trainings on the Istanbul protocol, Vienna, World Psychiatric Association Scientific Section on Sequels to Persecution and Torture, 2020 (e-book), p.4

zu klären, wie man unter Wahrung des Schutzes der Betroffenen und adäquatem Datenschutz der Gesundheitsdaten mit ihrem Einverständnis (,informed consent“) wichtige Informationen zu Folter in einem Land an internationale Einrichtungen wie an das Antifolterkomitee der Vereinten Nationen oder den Sonderberichterstatter zu Folter weitergeben könnte.

Ein besonderer Aspekt ist jener der Strafverfolgung von Täter/inne/n, die durch ihr eigenes Herkunftsland geschützt werden. Dies ist dadurch bedingt, dass die internationalen Gerichtshöfe derzeit noch zu wenige Staaten erfassen; einerseits, weil viele das Romstatut der internationalen Gerichtshöfe nicht unterzeichnet haben, andererseits, weil entsprechende Initiativen durch Vetomächte, oft durch die USA oder Russland, verhindert werden, was im Falle von Syrien besonders deutlich geworden ist.

Eine neue Initiative beschäftigt sich daher mit der Anwendung der „Universal Jurisdiction“-Strategien (in etwas unklarer historischer deutscher Übersetzung „Weltrechtspflege“), die es in vielen Staaten ermöglicht, eine Untersuchung schwerer Menschenrechtsverletzungen gegen Täter/innen in einem Drittland einzuleiten und diese in 
einigen Fällen, besonders, wenn sie das Territorium des entsprechenden Landes beispielsweise in Europa betreten, für die Durchführung eines Strafverfahrens zu inhaftieren. Auch hierfür ist eine adäquate Zeugenaussage ohne Retraumatisierung der Opfer sowie eine medizinische und psychologische Untersuchung zur Sicherung von Beweismitteln von wesentlicher Bedeutung. Das Istanbul-Protokoll wäre auch hier anzuwenden. Erste Erfolge sind dabei aus Deutschland zu verzeichnen (Human Rights WaTch 2014).

\section{Zusammenfassung und Perspektiven}

Die Umsetzung besonders wichtiger Menschenrechte wie der Schutz vor Folter und die Betreuung von Überlebenden und Angehörigen ist eine wesentliche interdisziplinäre Aufgabe, bei der Gesundheitsberufe, Jurist/inn/en, Kulturanthropolog/inn/en zusammenarbeiten und auch öffentlich die Interessen der Betroffenen unterstützen sollen. Wichtige Instrumente wie das Istanbul-Protokoll sollten vermehrt zur Anwendung kommen, um einerseits Betroffene zu schützen und zu unterstützen und andererseits zur Prävention gegen weitere Folteranwendung beizutragen. Diese wichtigen interdisziplinären Ansätze sollten, nicht zuletzt aufgrund der breiten Anwendung politischer Gewalt, auch vermehrt in die universitären Ausbildungen und die Fortbildungscurricula eingebunden werden. Während insgesamt international genügend Daten zu den schweren Langzeitfolgen von Folter und Krieg vorliegen, sind bestimmte Aspekte des Problems, wie die erweiterte Betreuung von Angehörigen, transitionale Justiz (,transitional justice“) und (auch psychologisch) therapeutisch wirksame Rechtsschritte (,therapeutic justice“) in Gebieten mit breit gefächerten Menschenrechtsverletzungen noch unzureichend gelöst und erforscht (Wenzel et al. 2015).

\section{Bibliographie}

Abu Suhaiban H., Grasser L.R. \& Javanbakht A. (2019), Mental Health of Refugees and Torture Survivors: A Critical Review of Prevalence, Predictors, and Integrated Care. International Journal of Environmental Research and Public Health, 16 (13).

Adaku A., Okello J., Lowry B., Kane J.C., Alderman S., Musisi S. et al. (2016), Mental health and psychosocial support for South Sudanese refugees in northern Uganda: a needs and resource assessment. Conflict and Health, 10 (1), p. 18.

Adelman A. (1995), Traumatic memory and the intergenerational transmission of Holocaust narratives. The Psychoanalytic Study of the Child, 50, pp. 343-67.

Adenauer H., Catani C., Gola H., Keil J., Ruf M., Schauer M. et al. (2011), Narrative exposure therapy for PTSD increases top-down processing of aversive stimuli - evidence from a randomized controlled treatment trial. BMC Neuroscience, 12, p. 127. 
Almli L.M., Fani N., Smith A.K. \& Ressler K.J. (2014), Genetic approaches to understanding post-traumatic stress disorder. International Journal of Neuropsychopharmacology, 17 (2), pp. 355-370.

Anton S., Tomanovic K., Mrdenovic S., Katinic K. \& Gugic D. (2011), Topic J. Influence of psychical trauma through transgenerational transfer on the development of traumatic reactions in women with diagnosed breast cancer. Collegium Antropologicum, 35 (3), pp. 673-680.

Arluke A., Lankford A. \& Madfis E. (2018), Harming animals and massacring humans: Characteristics of public mass and active shooters who abused animals. Behavioral Science \& the Law, 36 (6), pp. 739-751.

Asplund R. (1999), Sleep disorders in the elderly. Drugs \& Aging, 14 (2), pp. 91-103.

Betancourt T.S. (2008), Child soldiers: reintegration, pathways to recovery, and reflections from the field. Journal of Developmental and Behavioral Pediatrics, 29 (2), pp. 138-141.

Bohannon J. (2015), Scientific societies. Torture report prompts APA apology. Science, 349 (6245), pp. 221-222.

Bohleber W. (2007), Remembrance, trauma and collective memory: the battle for memory in psychoanalysis. International Journal of Psychoanalysis, 88 (Pt 2), pp. 329-352.

Bower H. (1994), The concentration camp syndrome. Australian and New Zealand Journal of Psychiatry, 28 (3), pp. 391-397.

Boyd J.W., LoCicero A., Malowney M., Aldis R. \& Marlin R.P. (2014), Failing ethics 101: psychologists, the U.S. military establishment, and human rights. International Journal of Health Services, 44 (3), pp. 615-625.

Bracken P.J., Giller J.E. \& Ssekiwanuka J.K. (1996), The rehabilitation of child soldiers: defining needs and appropriate responses. Medicine, Conflict and Survival, 12 (2), pp. 114-125.

Busch J., Hansen S.H. \& Hougen H.P. (2015), Geographical distribution of torture: An epidemiological study of torture reported by asylum applicants examined at the Department of Forensic Medicine, University of Copenhagen. Torture: Journal on Rehabilitation of Torture Victims and Prevention of Torture, 25 (2), pp. 12-21.

Chelala C. (1997), Doctor accused in Argentine grandmothers’ crusade. The Lancet, 349 (9046), p. 187.

CoRbin J.N. (2008), Returning home: resettlement of formerly abducted children in Northern Uganda. Disasters, 32 (2), pp. 316-335.

DE Wet E. (2004), The Prohibition of Torture as an International Norm of juscogens and Its Implications for National and Customary Law. European Journal of International Law, 15 (1), pp. 97-121.

DudAi R. (2006), Understanding perpetrators in genocides and mass atrocities. British Journal of Sociology, 57 (4), pp. 699-707.

Dyer O. (2016), Prosecution of US psychologists who ran CIA torture program moves a step closer. The British Medical Journal, 353, i2454.

Esala J.J. \& TaIng S. (2017), Testimony Therapy With Ritual: A Pilot Randomized Controlled Trial. Journal of Traumatic Stress, 30 (1), pp. 94-98.

Esparza M., Huttenbach H.R. \& Feierstein D. (2010), State violence and genocide in Latin America: the Cold War years. London, New York, Routledge. 
Fernandes P. \& Aiello Y. (2018), Breaking the silence through MANTRA: Empowering Tamil MAN survivors of torture and rape. Torture: Journal on Rehabilitation of Torture Victims and Prevention of Torture, 28 (3), pp. 14-29.

Fernandez M., Pissiota A., Frans O., von Knorring L., Fischer H. \& Fredrikson M. (2001), Brain function in a patient with torture related post-traumatic stress disorder before and after fluoxetine treatment: a positron emission tomography provocation study. Neuroscience Letters, 297 (2), pp. 101-104.

Frewer A., Furtmayr H.F., Krása K. \& Wenzel T. (2012), Istanbul-Protokoll. V\&R Unipress.

Furtmayr H. \& Frewer A. (2010), Documentation of torture and the Istanbul Protocol: applied medical ethics. Medicine, Health Care and Philosophy, 13 (3), pp. 279-286.

GRIFFIN R. (2017), The role of heroic doubling in ideologically motivated state and terrorist violence. International Review of Psychiatry, 29 (4), pp. 355-361.

Gruen A. (1992), Der Wahnsinn der Normalität. Stuttgart, DTV.

Halvorsen J.O. \& Stenmark H. (2010), Narrative exposure therapy for posttraumatic stress disorder in tortured refugees: a preliminary uncontrolled trial. Scandinavian Journal of Psychology, 51 (6), pp. 495-502.

Haritos-Fatouros M. (1988), The Official Torturer: A Learning Model for Obedience to the Authority of Violence. Journal of Applied Social Psychology, 18 (13), pp. 1107-1120.

Haslam S.A \& Reicher S. (2007), Beyond the banality of evil: three dynamics of an interactionist social psychology of tyranny. Personality and Social Psychology Bulletin, 33 (5), pp. 615622.

Helmchen H. \& Okasha A. (2000), From the Hawaii Declaration to the Declaration of Madrid. Acta Psychiatrica Scandinavia. Supplementum, 399, pp. 20-23.

Hensel-Dittmann D., Schauer M., Ruf M., Catani C., Odenwald M., Elbert T. et al. (2011), Treatment of traumatized victims of war and torture: a randomized controlled comparison of narrative exposure therapy and stress inoculation training. Psychotherapy and Psychosomatics, 80 (6), pp. 345-352.

Human Rights Watch (ed.) (2014), The Legal Framework for Universal Jurisdiction in Germany. New York, Human Rights Watch.

Izquierdo R., Makashvili N., Drozdek B. \& Wenzel T. (2019), Mental Health and Well-Being of the Staff Supporting Refugees: How to Deal with Risks? In: Wenzel T. \& Drozdek B. (eds.): An Uncertain Safety. New York, Springer, pp. 363-384.

Jawaid A., Roszkowski M. \& Mansuy I.M. (2018), Transgenerational Epigenetics of Traumatic Stress. Progress in Molecular Biology and Translational Science, 158, pp. 273-298.

Kellermann N.P. (2001), Psychopathology in children of Holocaust survivors: a review of the research literature. Israel Journal of Psychiatry and Related Sciences, 38 (1), pp. 36-46.

Kellermann N.P. (2013), Epigenetic transmission of Holocaust trauma: can nightmares be inherited? Israel Journal of Psychiatry and Related Sciences, 50 (1), pp. 33-39.

Kingston L. (2015), The Destruction of Identity: Cultural Genocide and Indigenous Peoples. Journal of Human Rights, 14.

KirmaYer L.J., Gone J.P. \& Moses J. (2014), Rethinking historical trauma. Transcultural Psychiatry, 51 (3), pp. 299-319.

Klasen F., Schrage J., Post M. \& Adam H. (2011), Guiltless guilty - trauma-related guilt and posttraumatic stress disorder in former Ugandan child soldiers. Praxis der Kinderpsychologie und Kinderpsychiatrie, 60 (2), pp. 125-142. 
Klasen F., Reissmann S., Voss C. \& Okello J. (2015), The guiltless guilty: trauma-related guilt and psychopathology in former Ugandan child soldiers. Child Psychiatry \& Human Development, 46 (2), pp. 180-193.

MacDonald B.K., Mummery C.J. \& Heaney D. (2001), Health needs of asylum seekers and refugees. Head injury needs to be taken into consideration in survivors of torture. The British Medical Journal, 323 (7306), p. 230.

McCoubrey H. (1995), Before “Geneva” Law: a British Surgeon in the Crimean War. International Review of the Red Cross, 304.

Mirzaei S., Kletecka-Pulker M. \& Wenzel T. (2019), Case report: Medical Researcher Imprisoned and Sentenced to Death during a Congress Visit. Clinical Case Reports International, 3, p. 1127.

Moon J.E. (2004), The death of distinctions: From 9/11 to Abu Ghraib. Politics and the Life Sciences, 23 (2), pp. 2-12.

Moreno A. \& Grodin, M.A. (2002), Torture and its neurological sequelae. Spinal Cord, 40 (5), pp. 213-223.

Musalo K. (1997), When Rights and Cultures Collide. Issues in Ethics, 8 (3).

North C.S., Suris A.M., Smit, R.P. \& King R.V. (2016), The evolution of PTSD criteria across editions of DSM. Annals of Clinical Psychiatry, 28 (3), pp. 197-208.

Olson B., Soldz S. \& Davis M. (2008), The ethics of interrogation and the American Psychological Association: a critique of policy and process. Philosophy, Ethics, and Humanities in Medicine, 3, p. 3.

PECKL P. (2007), [“The patient before was always healthy" - the "war trembler” in the mirror of military hospital patient records of German soldiers during First World War]. Praxis, Bern 1994; 96 (51-52), pp. 2075-2079.

Perez-Sales P., Jan den Otter J., Hardi L., Wenzel T., Diaconu G., Cors, G. et al. (2018), WPA Position Statement on Banning the Participation of Psychiatrists in the Interrogation of Detainees. World Psychiatry, 17 (2), pp. 237-238.

Peters, U.H. (1989), [Psychological sequelae of persecution. The survivor syndrome]. Fortschritte der Neurologie Psychiatrie, 57 (5), pp. 169-191.

Pham P.N., Vinck P. \& Stover E. (2009), Returning home: forced conscription, reintegration, and mental health status of former abductees of the Lord's Resistance Army in northern Uganda. BMC Psychiatry, 9, p. 23.

ReINEMAn J. (2011), Between the imaginary and the real: photographic portraits of mourning and of melancholia in Argentina. The International Journal of Psychoanalysis, 92 (5), pp. $1241-1261$.

RutAYisiRe T. \& Richters A. (2014), Everyday suffering outside prison walls: a legacy of community justice in post-genocide Rwanda. Social Science \& Medicine, 120, pp. 413420.

SAPOLSKY R.M. (2017), Behave: the biology of humans at our best and worst. New York, NY, Penguin Press.

Shrira A., Palgi Y., Ben-Ezra M. \& Shmotkin D. (2011), Transgenerational effects of trauma in midlife: Evidence for resilience and vulnerability in offspring of Holocaust survivors. Psychological Trauma: Theory, Research, Practice and Policy, 3 (4), pp. 394-402.

SINGH J.A. (2007), Treating war detainees and terror suspects: legal and ethical responsibilities of military physicians. Military Medicine, 172 (12 Suppl), pp. 15-21. 
Sмiтн L.A. (2017), The missing, the martyred and the disappeared: Global networks, technical intensification and the end of human rights genetics. Social Studies of Science, 47 (3), pp. 398-416.

Sorensen B. (1991), Why two torture committees? Journal of Medical Ethics, 17 Suppl, pp. $60-61$.

SORSCHER N. \& COHEN L.J. (1997), Trauma in children of Holocaust survivors: transgenerational effects. American Journal of Orthopsychiatry, 67 (3), pp. 493-500.

Stein D.J. (1998), Psychiatric aspects of the Truth and Reconciliation Commission in South Africa. The British Journal of Psychiatry, 173, pp. 455-457.

Tobin J.J. \& Friedman, J. (1983), Spirits, shamans, and nightmare death: survivor stress in a Hmong refugee. American Journal of Orthopsychiatry, 53 (3), pp. 439-448.

Tol W.A., Purgato M., Bass J.K., Galappatti A. \& Eaton W. (2015), Mental health and psychosocial support in humanitarian settings: a public mental health perspective. Epidemiology and Psychiatric Sciences, 24 (6), pp. 484-494.

Toprak S. (2009), The new face of terrorism in Turkey: actor unknown political murders. Journal of Forensic Sciences, 54 (6), pp. 1388-1392.

Verbitsky H. (1996), The flight: confessions of an Argentine dirty warrior. New York, New Press: Distributed by W.W. Norton.

Viller Hansen A.K., Sloth Hansen-Nord N., Smeir I., Engelkes-Heby L. \& Modvig J. (2017), Impact of NET on torture survivors in the MENA region. Torture, 27 (3), pp. 49-63.

Volkan V.D. (1997), Bloodlines: from ethnic pride to ethnic terrorism. $1^{\text {st }}$ ed. New York, Farrar, Straus and Giroux.

VOLKAN V.D. (2004), Blind trust: large groups and their leaders in times of crisis and terror. $1^{\text {st }}$ ed. Charlottesville, Va., Pitchstone Publishing.

VOLKAN V.D. (2006), Killing in the name of identity: a study of bloody conflicts. $1^{\text {st }}$ ed. Charlottesville, Va., Pitchstone Publishing.

WeIngARTEN K. (2004), Witnessing the effects of political violence in families: mechanisms of intergenerational transmission and clinical interventions. Journal of Marital and Family Therapy, 30 (1), pp. 45-59.

Welsh J. (1998), Truth and reconciliation ... and justice. The Lancet, 352 (9143), pp. 1852-1853.

Wenzel T. (2007), Torture. Current Opinion in Psychiatry, 20 (5), pp. 491-496.

Wenzel T., Sibitz I., KiefFer W. \& Strobl R. (1999), Capgras syndrome and functional psychosis in 2 survivors of torture. Psychopathology, 32 (4), pp. 203-206.

Wenzel T., Griengl H., Stompe T., Mirzaei S. \& Kieffer W. (2000), Psychological disorders in survivors of torture: exhaustion, impairment and depression. Psychopathology, 33 (6), pp. 292-296.

Wenzel T., Rushiti F., Aghani F., Diaconu G., Maxhuni B. \& Zitterl W. (2009), Suicidal ideation, post-traumatic stress and suicide statistics in Kosovo. An analysis five years after the war. Suicidal ideation in Kosovo. Torture, 19 (3), pp. 238-247.

Wenzel T., Özkalipci Ö. \& Frewer A. (2012), Das Istanbul-Protokoll - Perspektiven zur Entwicklung eines Standards der Vereinten Nationen. In: Frewer A., FurtmaYr H., KrásA K. und WenZeL T. (eds.): Istanbulprotokoll. Göttingen, V\&R Unipress, pp. 237-244.

Wenzel T., Frewer A. \& Mirzaei S. (2015), The DSM 5 and the Istanbul Protocol: Diagnosis of psychological sequels of torture. Torture. 25 (1), pp. 51-61. 
Wenzel T., Kienzler H. \& Wollmann A. (2015), Facing Violence - A Global Challenge. Psychiatric Clinics of North America, 38 (3), pp. 529-542.

Wenzel T., Mirzaei S. \& Nowak M. (2016), Assessment of sequelae of torture for refugees in host countries. The Lancet, 387 (10020), p. 746.

Wenzel T., den Otter J., Hardi L., Mirzaei S. \& Kletecka-Pulker M. (2019), Recent Cases of Women as Human Rights Defenders in Turkey and Near East. Archives of Women Health and Care, 2 (4), pp. 1-4.

Wenzel T., Drozdek B., Fu Chen A. \& Kletecka-Pulker M. (2019), The Significance of Intercultural Psychotherapy in Further Education and Professional Training. Intercultural Psychotherapy. New York, Springer.

Wenzel T., VölkL-Kernstock S., Wittek T. \& Baron D. (2019), Identifying Needs, Vulnerabilities and Resources in Refugee Persons and Groups. An Uncertain Safety. New York, Springer, pp. 51-99.

WiLson L.L. (1983), Torture, doctors and the World Medical Association. The Medical Journal of Australia, 2 (5), pp. 236-239.

Wilson R.A. (2000), Reconciliation and Revenge in Post-Apartheid South Africa. Rethinking Legal Pluralism and Human Rights. Current Anthropology, 41 (1), pp. 75-98.

WitZling D.R. (2015), Torture inefficiency long established. Science, 350 (6265), p. 1176.

Zandieh S., Bernt R., Knoll P., WenZel T., Hittmair K., Haller J. et al. (2016), Analysis of the Metabolic and Structural Brain Changes in Patients With Torture-Related Post-Traumatic Stress Disorder (TR-PTSD) Using (1)(8) F-FDG PET and MRI. Medicine (Baltimore), 95 (15), e3387. 